\title{
A SIMPLE PROOF OF HARDY'S INEQUALITY IN A LIMITING CASE
}

\section{FUTOSHI TAKAHASHI}

\begin{tabular}{|c|l|}
\hline Citation & OCAMI Preprint Series \\
\hline Issue Date & 2014 \\
\hline Type & Preprint \\
\hline Textversion & Author \\
\hline Rights & For personal use only. No other uses without permission. \\
\hline Relation & $\begin{array}{l}\text { This is a pre-print of an article published in Archiv der Mathematik. The } \\
\text { final authenticated version is available online at: } \\
\text { https://doi.org/10.1007/s00013-014-0711-8 }\end{array}$ \\
\hline
\end{tabular}

From: Osaka City University Advanced Mathematical Institute http://www.sci.osaka-cu.ac.jp/OCAMI/publication/preprint/preprint.html 


\title{
A SIMPLE PROOF OF HARDY'S INEQUALITY IN A LIMITING CASE
}

\author{
FUTOSHI TAKAHASHI
}

\begin{abstract}
In this short note, we provide a simple proof of Hardy's inequality in a limiting case. In the proof we do not need any rearrangement technique or the one-dimensional argument.
\end{abstract}

\section{INTRODUCTION}

Let $\Omega$ be a bounded domain in $\mathbb{R}^{N}$ with $0 \in \Omega$. The classical Hardy's inequality is of the form

$$
\left(\frac{N-p}{p}\right)^{p} \int_{\Omega} \frac{|u|^{p}}{|x|^{p}} d x \leq \int_{\Omega}|\nabla u|^{p} d x
$$

for all $u \in W_{0}^{1, p}(\Omega)$, where $N \geq 3$ and $1<p<N$. See [3] for its simple proof which uses the Fundamental Theorem of Calculus and Hölder's inequality only. It is well known that the constant $\left(\frac{N-p}{p}\right)^{p}$ is optimal and never attained in $W_{0}^{1, p}(\Omega)$.

For $p=N$, the inequality (1.1) loses its sense and instead of (1.1) the inequality

$$
\left(\frac{N-1}{N}\right)^{N} \int_{\Omega} \frac{|u(x)|^{N}}{|x|^{N}\left(\log \frac{R e}{|x|}\right)^{N}} d x \leq \int_{\Omega}|\nabla u|^{N} d x
$$

holds for all $u \in W_{0}^{1, N}(\Omega)$, where $R=\sup _{x \in \Omega}|x|$. Again, the constant $\left(\frac{N-1}{N}\right)^{N}$ is known to be optimal; see for example, [1], [2]. We call (1.2) as Hardy's inequality in a limiting case. Main aim of this short note is to provide a simple proof of Hardy's inequality in a limiting case. We do not need any rearrangement technique such as Polya-Szegö inequality for the spherical decreasing rearrangement, or a technical one-dimensional argument. Also our method can provide the sharper inequality treated in [8], [2], [9] and [7].

Date: August 25, 2014

1991 Mathematics Subject Classification. Primary 35A23, Secondary 26D15, 46E35. 
Theorem 1.1. Let $\Omega$ be a bounded domain in $\mathbb{R}^{N}, N \geq 2$ with $0 \in \Omega$. Let $g:(1,+\infty) \rightarrow \mathbb{R}$ be a $C^{2}$ function with the properties that

$$
g^{\prime}(s)<0, \quad g^{\prime \prime}(s)>0 \quad \text { for any } s>1
$$

and there exists $C>0$ such that

$$
\frac{\left(-g^{\prime}(s)\right)^{2(N-1)}}{\left(g^{\prime \prime}(s)\right)^{N-1}} \leq C \quad \text { for any } s>1 .
$$

Put $R=\sup _{x \in \Omega}|x|$. Then the inequality

$$
\begin{gathered}
\left(\frac{N-1}{N}\right)^{N} \int_{\Omega} \frac{|u(x)|^{N}}{|x|^{N}}\left(-g^{\prime}\left(\log \frac{R e}{|x|}\right)\right)^{N-2} g^{\prime \prime}\left(\log \frac{R e}{|x|}\right) d x \\
\leq \int_{\Omega} \frac{\left(-g^{\prime}\left(\log \frac{R e}{|x|}\right)\right)^{2(N-1)}}{\left(g^{\prime \prime}\left(\log \frac{R e}{|x|}\right)\right)^{N-1}}\left|\nabla u \cdot \frac{x}{|x|}\right|^{N} d x
\end{gathered}
$$

holds true for any $u \in W_{0}^{1, N}(\Omega)$.

Corollary 1.2. Let $\Omega$ be a bounded domain in $\mathbb{R}^{N}, N \geq 2$ with $0 \in \Omega$. Then the inequality

$$
\left(\frac{N-1}{N}\right)^{N} \int_{\Omega} \frac{|u(x)|^{N}}{|x|^{N}\left(\log \frac{R}{|x|}\right)^{N}} d x \leq \int_{\Omega}\left|\nabla u \cdot \frac{x}{|x|}\right|^{N} d x
$$

holds for all $u \in W_{0}^{1, N}(\Omega)$, where $R=\sup _{x \in \Omega}|x|$.

Note that, different from the function $\frac{1}{|x|^{N}\left(\log \frac{R e}{|x|}\right)^{N}}$ appeared in (1.2), the function $\frac{1}{|x|^{N}\left(\log \frac{R}{|x|}\right)^{N}}$ in (1.6) becomes unbounded when $|x| \sim 0$ and also $|x| \sim R$.

Corollary 1.3. Let $\Omega$ be a bounded domain in $\mathbb{R}^{N}, N \geq 2$ with $0 \in \Omega$. Let $\alpha>0$. Then the inequality

$$
\left(\frac{N-1}{N}\right)^{N} \alpha^{N} \int_{\Omega} \frac{|u(x)|^{N}}{|x|^{N-\alpha(N-1)}} d x \leq \int_{\Omega}|x|^{\alpha(N-1)}\left|\nabla u \cdot \frac{x}{|x|}\right|^{N} d x
$$

holds for all $u \in W_{0}^{1, N}(\Omega)$.

If we take $\alpha=\frac{N}{N-1}$ in (1.7), we have

$$
\int_{\Omega}|u(x)|^{N} d x \leq \int_{\Omega}|x|^{N}\left|\nabla u \cdot \frac{x}{|x|}\right|^{N} d x\left(\leq R^{N} \int_{\Omega}|\nabla u|^{N} d x\right),
$$

thus (1.7) can be seen as a generalization of Poincaré's inequality for $u \in$ $W_{0}^{1, N}(\Omega)$. 
The proof of Theorem 1.1 relies on the divergence theorem and Hölder's inequality. Similar "simple" approaches have been proposed in [10], [4], and [5], mainly to derive (1.1). Their proof uses the identity

$$
\operatorname{div}\left(\frac{x}{|x|^{\lambda}}\right)=\frac{N-\lambda}{|x|^{\lambda}} \text { for }|x| \neq 0, \lambda \in \mathbb{R}
$$

On the other hand, the identity

$$
\operatorname{div}\left(\frac{x}{|x|^{N}\left(\log \frac{R}{|x|}\right)^{N-1}}\right)=\frac{N-1}{|x|^{N}\left(\log \frac{R}{|x|}\right)^{N}} \text { for }|x| \neq 0, R
$$

will be the base of our proof. See the next section.

Another approach to the limiting case of Hardy's inequality, using the mean integral of a Schwarz symmetrization, has been done by Ioku; see [6]:Remark 1.4.

\section{Proof of Theorem.}

In this section, we prove Theorem 1.1. For $\varepsilon>0$ small, put

$$
R_{\varepsilon}=\sup _{x \in \Omega}\left(|x|^{2}+2 \varepsilon^{2}\right)^{1 / 2}, \quad X_{\varepsilon}(x)=\log \frac{R_{\varepsilon} e}{\left(|x|^{2}+\varepsilon^{2}\right)^{1 / 2}}
$$

and

$$
\psi_{\varepsilon}(x)=g\left(X_{\varepsilon}(x)\right) \in C^{2}(\bar{\Omega}) .
$$

We calculate

$$
\begin{aligned}
& \left|\nabla \psi_{\varepsilon}(x)\right|^{N-2} \nabla \psi_{\varepsilon}(x)=\left(-g^{\prime}\left(X_{\varepsilon}\right)\right)^{N-1}\left(\frac{|x|^{N-2} x}{\left(|x|^{2}+\varepsilon^{2}\right)^{N-1}}\right), \\
& \Delta_{N} \psi_{\varepsilon}(x)=\operatorname{div}\left(\left|\nabla \psi_{\varepsilon}(x)\right|^{N-2} \nabla \psi_{\varepsilon}(x)\right) \\
& =(N-1)\left(-g^{\prime}\left(X_{\varepsilon}\right)\right)^{N-2} g^{\prime \prime}\left(X_{\varepsilon}\right) \frac{|x|^{N}}{\left(|x|^{2}+\varepsilon^{2}\right)^{N}}+\left(-g^{\prime}\left(X_{\varepsilon}\right)\right)^{N-1} \frac{2(N-1) \varepsilon^{2}|x|^{N-2}}{\left(|x|^{2}+\varepsilon^{2}\right)^{N}},
\end{aligned}
$$

where we have used the assumption that $g^{\prime}(s)<0$. For $u \in W_{0}^{1, N}(\Omega)$, divergence theorem assures that

$$
\int_{\Omega}|u|^{N} \Delta_{N} \psi_{\varepsilon} d x=-\int_{\Omega} \nabla\left(|u|^{N}\right) \cdot\left|\nabla \psi_{\varepsilon}\right|^{N-2} \nabla \psi_{\varepsilon} d x
$$


The RHS of (2.2) is estimated from above as

$$
\begin{aligned}
& \mid \text { RHS of }(2.2)|=| N \int_{\Omega}|u|^{N-2} u \nabla u \cdot\left|\nabla \psi_{\varepsilon}\right|^{N-2} \nabla \psi_{\varepsilon} d x \mid \\
& =\left.\left|N \int_{\Omega}\right| u\right|^{N-2} u\left(-g^{\prime}\left(X_{\varepsilon}\right)\right)^{N-1}\left(\frac{|x|^{N-2} x \cdot \nabla u}{\left(|x|^{2}+\varepsilon^{2}\right)^{N-1}}\right) d x \mid \\
& \leq N \int_{\Omega}|u|^{N-1}\left(-g^{\prime}\left(X_{\varepsilon}\right)\right)^{N-1}\left(\frac{|x|^{N-2}|x \cdot \nabla u|}{\left(|x|^{2}+\varepsilon^{2}\right)^{N-1}}\right) d x \\
& \leq N\left(\int_{\Omega} \frac{|u|^{N}|x|^{N}}{\left(|x|^{2}+\varepsilon^{2}\right)^{N}}\left(-g^{\prime}\left(X_{\varepsilon}\right)\right)^{N-2} g^{\prime \prime}\left(X_{\varepsilon}\right) d x\right)^{\frac{N-1}{N}} \times \\
& \times\left(\int_{\Omega}\left(-g^{\prime}\left(X_{\varepsilon}\right)\right)^{2(N-1)}\left(g^{\prime \prime}\left(X_{\varepsilon}\right)\right)^{-(N-1)}\left|\nabla u \cdot \frac{x}{|x|}\right|^{N} d x\right)^{\frac{1}{N}},
\end{aligned}
$$

where we have used Hölder's inequality. On the other hand, the LHS of (2.2) is estimated from below as

$\mid$ LHS of (2.2)|

$$
\begin{aligned}
& =(N-1) \int_{\Omega}|u|^{N}\left\{\left(-g^{\prime}\left(X_{\varepsilon}\right)\right)^{N-2} g^{\prime \prime}\left(X_{\varepsilon}\right) \frac{|x|^{N}}{\left(|x|^{2}+\varepsilon^{2}\right)^{N}}+\left(-g^{\prime}\left(X_{\varepsilon}\right)\right)^{N-1} \frac{2 \varepsilon^{2}|x|^{N-2}}{\left(|x|^{2}+\varepsilon^{2}\right)^{N}}\right\} d x \\
& \geq(N-1) \int_{\Omega}|u|^{N}\left(-g^{\prime}\left(X_{\varepsilon}\right)\right)^{N-2} g^{\prime \prime}\left(X_{\varepsilon}\right) \frac{|x|^{N}}{\left(|x|^{2}+\varepsilon^{2}\right)^{N}} d x .
\end{aligned}
$$

Thus we have

$$
\begin{aligned}
(N-1) & \int_{\Omega} \frac{|u|^{N}|x|^{N}}{\left(|x|^{2}+\varepsilon^{2}\right)^{N}}\left(-g^{\prime}\left(X_{\varepsilon}\right)\right)^{N-2} g^{\prime \prime}\left(X_{\varepsilon}\right) d x \\
\leq & N\left(\int_{\Omega} \frac{|u|^{N}|x|^{N}}{\left(|x|^{2}+\varepsilon^{2}\right)^{N}}\left(-g^{\prime}\left(X_{\varepsilon}\right)\right)^{N-2} g^{\prime \prime}\left(X_{\varepsilon}\right) d x\right)^{\frac{N-1}{N}} \times \\
& \times\left(\int_{\Omega}\left(-g^{\prime}\left(X_{\varepsilon}\right)\right)^{2(N-1)}\left(g^{\prime \prime}\left(X_{\varepsilon}\right)\right)^{-(N-1)}\left|\nabla u \cdot \frac{x}{|x|}\right|^{N} d x\right)^{\frac{1}{N}},
\end{aligned}
$$

which implies

$$
\begin{gathered}
\left(\frac{N-1}{N}\right)^{N} \int_{\Omega}|u|^{N}\left(-g^{\prime}\left(X_{\varepsilon}\right)\right)^{N-2} g^{\prime \prime}\left(X_{\varepsilon}\right) \frac{|x|^{N}}{\left(|x|^{2}+\varepsilon^{2}\right)^{N}} d x \\
\leq \int_{\Omega} \frac{\left(-g^{\prime}\left(X_{\varepsilon}\right)\right)^{2(N-1)}}{\left(g^{\prime \prime}\left(X_{\varepsilon}\right)\right)^{N-1}}\left|\nabla u \cdot \frac{x}{|x|}\right|^{N} d x
\end{gathered}
$$

Finally, we let $\varepsilon \rightarrow 0$ in the both sides of the above inequality. Note that $R_{\varepsilon} \rightarrow R$ and $X_{\varepsilon} \rightarrow \log \left(\frac{R e}{|x|}\right)$ a.e. $x \in \Omega$ as $\varepsilon \rightarrow 0$. By using Fatou's 
lemma in the LHS and the Lebesgue dominated convergence theorem with the assumption (1.4) in the RHS, we conclude (1.5) holds.

\section{Proof of Corollary 1.2, 1.3.}

Proof. For the proof of Corollary 1.2, let $g(s)=-\log (s-1)$ for $s>1$. Then we see $g^{\prime}(s)=-\frac{1}{s-1}<0, g^{\prime \prime}(s)=\frac{1}{(s-1)^{2}}>0$, and

$$
\frac{\left(-g^{\prime}(s)\right)^{2(N-1)}}{\left(g^{\prime \prime}(s)\right)^{N-1}}=\frac{\left(\frac{1}{(s-1)}\right)^{2(N-1)}}{\left(\frac{1}{(s-1)^{2}}\right)^{N-1}}=1 \quad \text { for any } s>1 .
$$

Thus the assumptions (1.3),(1.4) are satisfied. Inserting

$$
g^{\prime}\left(\log \frac{R e}{|x|}\right)=\frac{-1}{\log \frac{R}{|x|}}, \quad g^{\prime \prime}\left(\log \frac{R e}{|x|}\right)=\frac{1}{\left(\log \frac{R}{|x|}\right)^{2}}
$$

into (1.5), we obtain (1.6).

For Corollary 1.3, take $g(s)=e^{-\alpha s}$ for $s>1$. We easily see that

$$
\frac{\left(-g^{\prime}(s)\right)^{2(N-1)}}{\left(g^{\prime \prime}(s)\right)^{N-1}}=e^{-(N-1) \alpha s} \leq 1 \quad \text { for any } s>1
$$

and

$$
g^{\prime}\left(\log \frac{R e}{|x|}\right)=-\alpha\left(\frac{|x|}{R e}\right)^{\alpha}, \quad g^{\prime \prime}\left(\log \frac{R e}{|x|}\right)=\alpha^{2}\left(\frac{|x|}{R e}\right)^{\alpha} .
$$

Using these and (1.5), we have (1.7).

Acknowledgement. Part of this work was done when the author visited KAIST and KIAS in Korea, August, 2014. He thanks both institutes for its support and hospitality. The author was also supported by JSPS Grant-inAid for Scientific Research (B), No. 23340038, and JSPS Grant-in-Aid for Challenging Exploratory Research, No. 24654043.

\section{REFERENCES}

1. Adimurthi, N. Chaudhuri, and M. Ramaswamy: An improved Hardy-Sobolev inequality and its application, Proc. Amer. Math. Soc., 130 no.2, 489-505, (2001)

2. Adimurthi, and K. Sandeep: Existence and non-existence of the first eigenvalue of the perturbed Hardy-Sobolev operator, Proc. Roy. Soc. Edinburgh Sect. A, 132 no.5, 1021-1043, (2002)

3. J.P. García Azorero, and I. Peral Alonso: Hardy inequalities and some critical elliptic and parabolic problems, J. Differential Equations. 144 441-476, (1998)

4. D. G. Costa: Some new and short proofs for a class of Caffarelli-Kohn-Nirenberg type inequalities, J. Math. Anal. Appl., 337 311-317, (2008)

5. Y. Di, L. Jiang, S. Shen, and Y. Jin: A note on a class of Hardy-Rellich type inequalities, J. Inequal. Appl., 2013:84 1-6, (2013)

6. N. Ioku: Sharp Sobolev inequalities in Lorenz spaces for a mean oscillation, J. Funct. Anal., 266, 2914-2958, (2014)

7. N. Ioku, and M. Ishiwata: talk at Yamagata kick off seminar, 15 July, (2013) 
8. O. A. Ladyzhenskaya: The mathematical theory of viscous incompressible flow, Second edition, revised and enlarged. Mathematics and its Applications, Vol. 2 Gordon and Breach, Science Publishers, New York-London-Peris, (1969)

9. S. Machihara, T. Ozawa, and H. Wadade: Hardy type inequalities on balls, Tohoku Math. J. (2), 65 no.3, 321-330, (2013)

10. E. Mitidieri: A simple approach to Hardy inequalities, Mathematical Notes,. 67, no.4, $563-572,(2000)$

Department of Mathematics, Osaka City University \& OCAMi, SumiyoshiKU, OSAKA, 558-8585, JAPAN

E-mail address: futoshi@sci.osaka-cu.ac.jp 